\title{
Up-scaling the power of pulsed single frequency fibre amplifiers for coherent LIDAR applications
}

\author{
François Gustave ${ }^{1}$, Pierre Bourdon ${ }^{1}$, Julien Le Gouët ${ }^{1}$, Anne Durécu ${ }^{1}$, Laurent Lombard ${ }^{1}$, \\ Hermence Jacqmin ${ }^{1}$, Agnès Dolfi-Bouteyre ${ }^{1}$ \\ ${ }^{I}$ DOTA, ONERA, Université Paris Saclay, F-91123 Palaiseau - France
}

In optical sensing applications, the laser sources are strongly constrained by the sensing system requirements. In the case of coherent LIDARs, that measure wind speed thanks to the Doppler shift (by means of a coherent detection), highly coherent high power pulsed lasers with good output beam quality are necessary to guarantee a proper measurement over long distances. The pulsed emission ensures the spatial resolution along the line of sight whereas the high coherence accounts for the wind speed resolution (measured in the frequency domain). Thus, the challenge when designing laser sources for such instruments resides in the difficulty of improving the output power while not altering the other characteristics.

Fibre lasers and amplifiers have proven their interest in high power laser systems thanks to their compactness and their stability against temperature and mechanical fluctuations. The increase of emitted optical power is currently limited by nonlinear effects due to the intrinsically high confinement of the optical field in fibres. Among all the nonlinear effects, the Stimulated Brillouin Scattering (SBS) predominates in the case of single frequency or narrow spectrum optical fields.

In order to increase the power of single frequency fibre lasers, many developments have been done in the past decades to mitigate SBS. We can class the different SBS mitigation techniques in two categories depending on whether they act on the optical field properties or on the optical fibre itself. All the techniques belonging to the first category are based on an active control of the phase or the frequency of the incident optical wave, in order to spread the incident energy in the spectral domain. In the second category, we can maximize the core radius of the fibre, minimize the fibre length, apply temperature gradients, dopant concentrations gradients or strain gradients to broaden the SBS spectrum in a controlled manner and thus lower its effective gain.
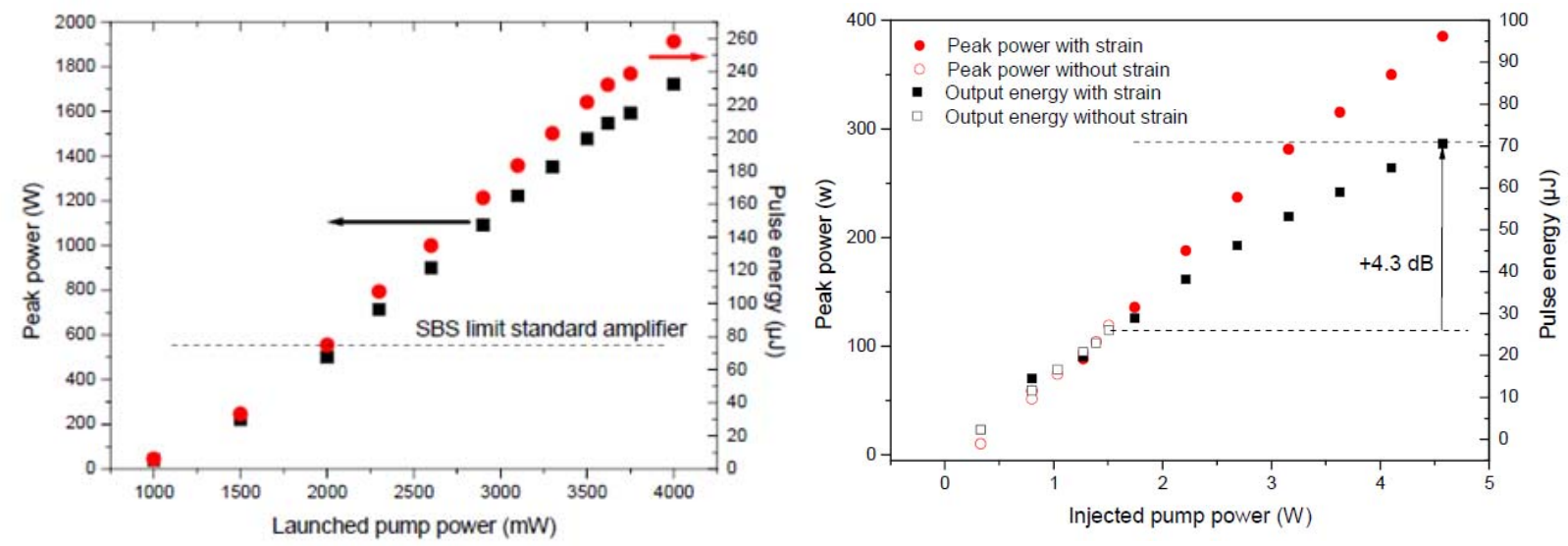

Fig. 1 Experimental output power and pulse energy of two realizations of single frequency pulsed fibre amplifiers. Left : Erbium doped amplifier at $1580 \mathrm{~nm}$ with tensile strain gradient on LMA active fibre. Right : ErYb co-doped single mode active fibre at $1545 \mathrm{~nm}$ with compressive strain gradient.

We review here the different techniques we use to mitigate the SBS in pulsed single frequency fibre amplifiers and discuss their conceptual and technical limitations. Among all the existing mitigation techniques, we present here different realizations of fibre amplifiers designed for coherent LIDAR systems, based on strain gradients [1] and specifically designed highly doped and large core active fibres [2].

Figure 1 presents the typical achievements obtained with our patented tensile and compressive strain gradients techniques when applied to active fibers. We are able to increase the output power by a factor 2 to 3 while keeping a high beam quality on a highly coherent laser emission. We emphasize the robustness of such developments thanks to the absence of active devices that make them suitable for embedded sensing applications.

\section{References}

[1] G. Canat, S. Makon-Makon, L. Lombard, J. Le Gouët, A. Durécu, "High peak power Erbium-Ytterbium single frequency pulsed fiber amplifier using a compressive strain gradient". SM4Q.6, CLEO (2016)

[2] A. Durécu, P. Bourdon, F. Gustave, H. Jacqmin, J. Le Gouët, and L. Lombard, "High Peak Power Single-Frequency Amplifier based on a Er-Yb Doped Polarization Maintaining LMA Fiber”. JTh2A.120, CLEO (2017) 